\title{
Calponin 1 Serum Level A Biological Marker for Preterm Labor Predictability
}

\author{
Mohamed Ibrahim Taema ${ }^{1 *}$ and Rania Ali Ammar Abd El Hakim² \\ ${ }^{1}$ Lecturer of obstetrics and gynecology, Ain Shams University, Egypt \\ ${ }^{2}$ Assistant Professor of clinical and chemical pathology, Ain Shams University, Egypt
}

Received: 阱 August 23, 2018; Published: 眥 August 29, 2018

*Corresponding author: Mohamed Ibrahim Taema, Lecturer of obstetrics and gynecology, Ain Shams University, Egypt

\section{Abstract}

Aim: To evaluate and assess the value of measuring calponin 1 serum level in maternal circulation as a predictability tool for preterm labor within 1 week in cases affected with threatened preterm delivery.

Methodology: cases clinically presenting at 24-34 gestational weeks with threatened preterm labor undergone sample collection for measuring calponin 1 serum level simultaneously with cervical length assessment. With follow up performed in a prospective manner till time of birth assessment and perinatal clinical outcomes of recruited subjects have been collected and tabulated for analysis.

Results: One hundred forty-seven patients affected by threatened preterm labor symptoms, between 24| and 33|+ 6gestational weeks have been enrolled in the research study. 71 cases $(48.3 \%)$ delivered within 1 week from admission (group-A) and the remaining 76 cases (51.7\%) delivered after 1 week from admission (group-B). Statistical correlations between the time interval between admission and delivery and cervical length\& calponin- 1 at admissions were significantly positive $(0.387, p<0.001) \&$ significantly negative $(-0.470, \mathrm{p}<0.001)$ respectively while it was between cervical length and calponin- 1 at admissions significantly negative $(-0.413, \mathrm{p}<0.001)$. Cervical length and calponin-1 at admissions had moderate Diagnostic performance in predicting delivery within 1 week from admission that not markedly increased if having any of them or by combination in a regression equation.

Conclusion: measuring and evaluating calponin 1 level in maternal serum could be a useful biological marker in short-range time period for predictability of preterm delivery within patients affected by threatened preterm delivery clinical presentation, besides cervical length dimension measuring.

\section{Introduction}

Preterm birth is featured and defined as delivery before 37 gestational weeks global occurrence in 15 million neonates and represents around $11 \%$ of all living births. The threatened preterm delivery has an incidence around two to three times greater between 24 and 34 gestational weeks than the 34-37 gestational weeks. The bulk of cases with preterm delivery clinical presentation before 32 gestational weeks are referred to a tertiary hospital and managed with tocolytic agents e.g, MgSO4 and lung maturity enhancing agent such as corticosteroids. On the other hand, around $80 \%$ of these patients would not give birth within 1 week after hospital admission, and 50 percent of those gestations deliver at term. For that reason, several patients are needlessly referred and exposed to the possible side effects of tocolytic drugs and corticosteroids for both mother and fetus [1]. The usefulness of forecasting those patients that will not give birth in the short period of time (within 1 week) is necessary consecutively to avoid

over management and reduce the costs of needless admissions and management protocol usage. Alternatively, the precise recognition of this form of symptomatic cases with preterm delivery symptoms aids to uncover those that require emergency interventional mode of management and referral to a tertiary level of care hospital for the aim to reduce the possible perinatal risks when preterm birth occurs [2]. Various researchers investigated biological markers and clinical approaches for predictability of preterm birth; yet, it still a difficult issue to evaluate this prediction in cases with threatened preterm labor. Prior research studies displayed that cervical length dimension measuring and fetal fibronectin testing could aid to identify patients that would not clinically benefit from tocolytic agent implementation. Numerous research systematic reviews priorly performed have displayed and revealed that the cervical length dimension more than $25 \mathrm{~mm}$ and a cut off value of $0.050 \mathrm{mg} /$ $\mathrm{mL}$ of fetal fibronectin are valuable as negative predictive indices for preterm delivery within 1 week [3]. 
Various researchers mentioned that calponin -1 protein, an actin filament-linked regulatory type of protein with a molecular weight of 34-37 kDa (292-330 amino acids), acting at molecular and cellular level as an inhibitor of actin-activated myosin ATPase in smooth muscles and non-muscular type of cells. Smooth muscle contractions are integrated in action chiefly by the reversible phosphorylation action of myosin light chain, being triggered by a rise in sarcoplasmic-free calcium cellular concentration [4]. Alternatively, these contractions could be organized and ordered by different signal transduction molecular and cellular action pathways, e.g the thin filament-linked calponin 1 protein. Our research considered that calponin 1 protein, which is featured as a smooth muscle troponin-like form of protein, could clinically serve as a biological marker for precise predictability of the time of birth in threatened preterm delivery case scenario. This research approached consideration and hypothesis is based mainly on the fact that calponin 1 , protein is the most copious and definite protein in smooth muscle tissue. In addition, all through the second and third gestational trimesters, the uterus contain the largest quantity of smooth muscle and calponin protein [5]. Additionally, uterine contractions causing the delivery process to occur Could cause myometrial damage that could lead to raised calponin 1 levels in maternal serum [6].

\section{Aim of the Work}

The aim of the recent research study conducted by our research is to settle on whether calponin 1 level in maternal serum could be used as a biological marker for prediction of labor within 1 week in gestations affected by threatened preterm labor between 24 and 34 gestational weeks. Additionally, we assessed whether the maternal serum calponin 1 level, if integrated to the cervical length dimension, enhance the short-term predictability of preterm delivery in symptomatic patients.

\section{Methodology}

This is a prospective research study performed at United Doctors Hospital, KSA, between December 2015 and December 2017. Local Ethics Committee of the hospital have approved the study and informed written consent was obtained from recruited subjects. One hundred forty-seven cases affected by threatened preterm labor symptoms, between 24] and 33|+ 6gestational weeks have been enrolled in the research study. 71cases (48.3\%) delivered within 1 week from admission (group-A) and the remaining 76 cases $(51.7 \%)$ delivered after 1 week from admission (group-B). twenty-three cases have been excluded from the study some did not meet the inclusive criteria and others have refused the participation in the research study. Exclusion criteria: gestations $>34$ weeks, multifetal gestations, preterm premature rupture of membranes, antepartum hemorrhage, fetal congenital malformations, hypertension, gestational DM, previous or consequent cerclage, medical and obstetric associated morbidities. Clinical diagnosis of threatened preterm delivery was established due to the occurrence of regular uterine contractions at $8 \mathrm{~min}$ intervals known by abdominal examination and tocography, either with or without occurrence of cervical changes.

Maternal blood sampling was obtained from Cases before start of medication (betamethasone and tocolytic agents. calponin 1 serum level was assayed using enzyme-linked immune sorbent assay (ELISA). All cases had cervical length assessment by transvaginal ultrasound scan. All cases were admitted and advised for bed rest. Initially managed with hydration. If persistence of uterine contractions has been observed or progressive cervical changes existed for at least $2 \mathrm{~h}$ after intravenous hydration, tocolytic management was commenced. A calcium-channel blocker agent, nifedipine (10-30mg oral daily) and 17-alpha-hydroxyprogesterone caproate (170HP-C, 250mg, i.m) were prescribed once a week until 36 gestational weeks or until the occurrence of preterm delivery, stoppage of tocolysis $48 \mathrm{~h}$ after the start of corticosteroids dose and we did not implement tocolytic agents and maternal corticosteroids after 34 weeks of gestation. Mode and time of birth was clinically decided according to case scenario, and cesarean delivery was performed for solid obstetric indications only. Clinical follow up of maternal and fetal outcomes were recorded in a prospective manner till the time of delivery. The gestational age at time of hospitalization, gestational age at birth, time interval from hospitalization till birth, neonatal birth weight, Apgar scoring and neonatal ICU admission of recruited cases were recorded.

The primary research outcome of interest was birth within 1 week after hospitalization. The cases were categorized into two divisions. Cases that delivered within 1 week after hospitalization, as research group A, and cases that gave birth after 1 week from hospitalization, as research group B. Descriptive statistical analysis for investigated variables was displayed as mean and standard deviation. Continuous research variables are put in comparison within the 2 groups using Student's t-test. A Chi-square test was used to examine the association between categorical variables. ROC statistical analysis has been applied to assess the predictability cut off value of maternal calponin 1 serum level. The sensitivity, specificity, positive predictive values, negative predictive values and likelihood ratios were calculated. Statistical significance levels were considered as 5\%. The SPSS statistical program was used for all statistical calculations.

\section{Results}

This cohort research study- a cohort of 147 patients- had completed a follow up from admission till delivery, amongst 71 (48.3\%) delivered within 1 week from admission (group-A) and the remaining $76(51.7 \%)$ delivered after 1 week from admission (group-B) (Figures $1 \& 2$ ) and (Table 1). Correlations between Time interval between admission and delivery and cervical length\& calponin-1 at admissions were statistically significantly positive (0.387, $\mathrm{p}<0.001)$ \&statistically significantly negative $(-0.470$, $\mathrm{p}<0.001$ ) (Figure 3) consecutively while it was between cervical length and calponin-1 at admissions statistically significantly negative $(-0.413, \mathrm{p}<0.001)$ (Figure 3$)$ and (Table 2).The significant 
equation to predict delivery within 1 week from admission is 1.062* Calponin-1 (ng/mL) - -0.122 Cervical length (mm) the optimum cutoff is showed in diagnostic performance (Table 3) and ( Figure 4) show that: cervical length and calponin-1 at admissions had moderate Diagnostic performance in predicting delivery within 1 week from admission that not markedly increased if having any of them or by combination in a regression equation.

Table 1: Comparison according to time.

\begin{tabular}{|c|c|c|c|c|c|}
\hline \multicolumn{2}{|c|}{ Variables } & $\begin{array}{c}\text { Total } \\
(\mathrm{N}=147)\end{array}$ & $\begin{array}{l}\text { Group-A } \\
(\mathrm{N}=71)\end{array}$ & $\begin{array}{l}\text { Group-B } \\
(\mathrm{N}=76)\end{array}$ & P-value \\
\hline \multicolumn{2}{|c|}{ Age (years)s } & $27.8 \pm 4.0$ & $28.1 \pm 4.2$ & $27.6 \pm 3.8$ & $\wedge 0.457$ \\
\hline \multicolumn{2}{|c|}{ BMI $\left(\mathrm{kg} / \mathrm{m}^{2}\right)$} & $26.9 \pm 2.4$ & $26.8 \pm 2.6$ & $27.0 \pm 2.2$ & $\wedge 0.577$ \\
\hline \multirow{2}{*}{ Parity } & Primiparous & $37(25.2 \%)$ & $17(23.9 \%)$ & $20(26.3 \%)$ & $\# 0.741$ \\
\hline & Multiparous & $110(74.8 \%)$ & $54(76.1 \%)$ & $56(73.7 \%)$ & \\
\hline \multicolumn{2}{|c|}{ Parity number } & $1.4 \pm 1.0$ & $1.5 \pm 1.1$ & $1.2 \pm 0.9$ & $\wedge 0.058$ \\
\hline \multicolumn{2}{|c|}{ Gravidity number } & $1.6 \pm 1.2$ & $1.6 \pm 1.2$ & $1.6 \pm 1.1$ & $\wedge 0.839$ \\
\hline \multicolumn{2}{|c|}{ Previous preterm delivery } & $16(10.9 \%)$ & $8(11.3 \%)$ & $8(10.5 \%)$ & $\# 0.885$ \\
\hline \multicolumn{2}{|c|}{ GA at admission (weeks) } & $29.0 \pm 1.9$ & $28.8 \pm 1.9$ & $29.1 \pm 1.9$ & $\wedge 0.307$ \\
\hline \multicolumn{2}{|c|}{$\mathrm{WBC}$ at admission $\left(\mathrm{x} 10^{3} / \mathrm{mL}\right)$} & $12.3 \pm 2.2$ & $12.2 \pm 2.1$ & $12.4 \pm 2.4$ & $\wedge 0.622$ \\
\hline \multicolumn{2}{|c|}{ CRP at admission (mg/dL) } & $4.9 \pm 2.0$ & $5.4 \pm 2.1$ & $4.5 \pm 1.8$ & ${ }^{\wedge} 0.006^{*}$ \\
\hline \multicolumn{2}{|c|}{ Calponin-1 at admission (ng/mL) } & $2.7 \pm 1.3$ & $3.3 \pm 1.1$ & $2.1 \pm 1.1$ & ${ }^{\wedge}<0.001^{*}$ \\
\hline \multicolumn{2}{|c|}{ Cervical length at admission (mm) } & $24.8 \pm 5.7$ & $21.3 \pm 4.9$ & $28.1 \pm 4.1$ & ${ }^{\wedge}<0.001^{*}$ \\
\hline \multirow{2}{*}{ Mode of delivery } & Vaginal & $53(36.1 \%)$ & $18(25.4 \%)$ & $35(46.1 \%)$ & \#0.009* \\
\hline & Cesarean & $94(63.9 \%)$ & $53(74.6 \%)$ & $41(53.9 \%)$ & \\
\hline \multicolumn{2}{|c|}{$\begin{array}{l}\text { Time interval between admission and delivery } \\
\text { (days) }\end{array}$} & $18.6 \pm 18.3$ & $4.4 \pm 1.3$ & $31.8 \pm 16.8$ & ${ }^{\wedge}<0.001^{*}$ \\
\hline \multicolumn{2}{|c|}{ GA at delivery (weeks) } & $31.7 \pm 3.3$ & $29.6 \pm 1.9$ & $33.7 \pm 3.0$ & ${ }^{\wedge}<0.001^{*}$ \\
\hline \multicolumn{2}{|c|}{ Birth weight (kg) } & $2.4 \pm 0.6$ & $2.1 \pm 0.4$ & $2.8 \pm 0.5$ & ${ }^{\wedge}<0.001^{*}$ \\
\hline \multicolumn{2}{|c|}{ APGAR1 } & $6.0 \pm 1.4$ & $5.1 \pm 1.0$ & $6.9 \pm 1.2$ & ${ }^{\wedge}<0.001^{*}$ \\
\hline \multicolumn{2}{|c|}{ APGAR5 } & $7.5 \pm 1.6$ & $6.4 \pm 1.2$ & $8.6 \pm 1.2$ & ${ }^{\wedge}<0.001^{*}$ \\
\hline \multicolumn{2}{|c|}{ NICU admission } & $34(23.1 \%)$ & $31(43.7 \%)$ & $3(3.9 \%)$ & $\#<0.001^{*}$ \\
\hline
\end{tabular}

${ }^{\wedge}$ Independent t-test, \#Chi square test, *Significant, GA: Gestational age, BMI: body mass index; CRP: C-Reactive Protein; NICU: Neonatal Intensive Care Unit; WBC: White Blood Cell count

Table 2: Logistic regression model for cervical length and calponin-1 at admissions to predict delivery within 1 week from admission.

\begin{tabular}{|c|c|c|c|c|}
\hline Factors & $\boldsymbol{\beta}$ & SE & P-value & OR (95\% CI) \\
\hline Calponin-1 $(\mathrm{ng} / \mathrm{mL})$ & 1.062 & 0.168 & $<0.001^{*}$ & $2.893(2.082-4.019)$ \\
\hline Cervical length $(\mathrm{mm})$ & -0.122 & 0.019 & $<0.001^{*}$ & $0.885(0.852-0.919)$ \\
\hline
\end{tabular}

$\beta$ : Regression coefficient, SE: Standard error, OR: Odds ratio, CI: Confidence interval, *Significant

Table 3: Diagnostic performance of cervical length and calponin-1 at admissions to predict delivery within 1 week from admission.

\begin{tabular}{|c|c|c|c|c|}
\hline Characteristics & Calponin-1 & Cervical length & $\begin{array}{c}\text { Any of Calponin-1 } \mathbf{2 . 4} \\
\mathbf{n g} / \mathbf{m L} \text { or Cervical length } \\
\mathbf{\leq 2 4 . 0}\end{array}$ & Regression equation \\
\hline AUC & 0.798 & 0.835 & & 0.864 \\
\hline SE & 0.037 & 0.032 & & $<.03$ \\
\hline P-value & $<0.001^{*}$ & $<0.001^{*}$ & $0.001^{*}$ \\
\hline 95\% CI & $0.726-0.870$ & $0.772-0.899$ & & $0.805-0.922$ \\
\hline Optimum cutoff & $\geq 2.4 \mathrm{ng} / \mathrm{mL}$ & $\leq 24.0 \mathrm{~mm}$ & & $\geq 0.27$ \\
\hline Sensitivity & $78.90 \%$ & $69.00 \%$ & & $90.10 \%$ \\
\hline
\end{tabular}




\begin{tabular}{|c|c|c|c|c|}
\hline Specificity & $71.10 \%$ & $80.30 \%$ & $64.50 \%$ & $72.40 \%$ \\
\hline Diagnostic accuracy & $74.80 \%$ & $74.80 \%$ & $74.80 \%$ & $81.00 \%$ \\
\hline Youden's index & $49.90 \%$ & $49.30 \%$ & $50.40 \%$ & $62.50 \%$ \\
\hline Positive predictive value & $71.80 \%$ & $76.60 \%$ & $69.30 \%$ & $75.30 \%$ \\
\hline Negative predictive value & $78.30 \%$ & $73.50 \%$ & $83.10 \%$ & $88.70 \%$ \\
\hline Likelihood ratio positive & 2.72 & 3.5 & 2.42 & 3.26 \\
\hline Likelihood ratio negative & 0.3 & 0.39 & 0.22 & 0.14 \\
\hline Likelihood ratio & 9.16 & 9.06 & 11.07 & 23.95 \\
\hline & & & & \\
\hline
\end{tabular}

AUC: Area under curve, SE: Standard error, CI: Confidence interval, *Significant, NPV: negative predictive value; PPV: positive predictive value

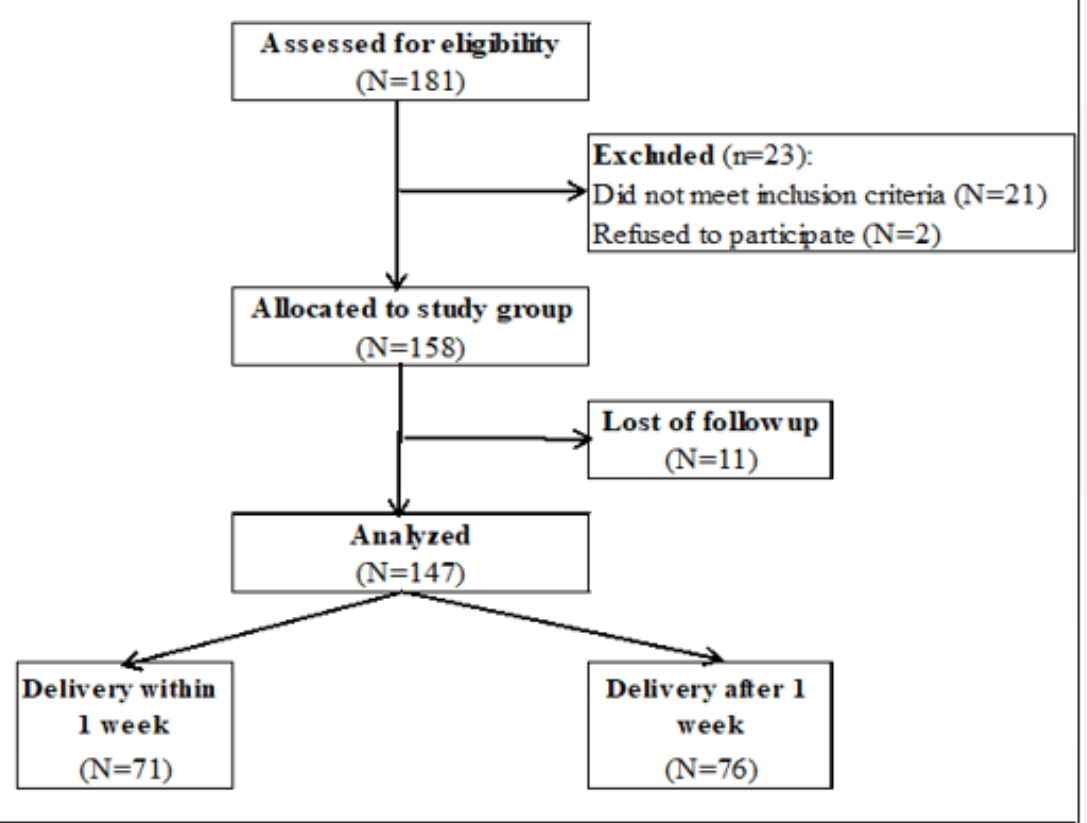

Figure 1: Patients' flow chart.

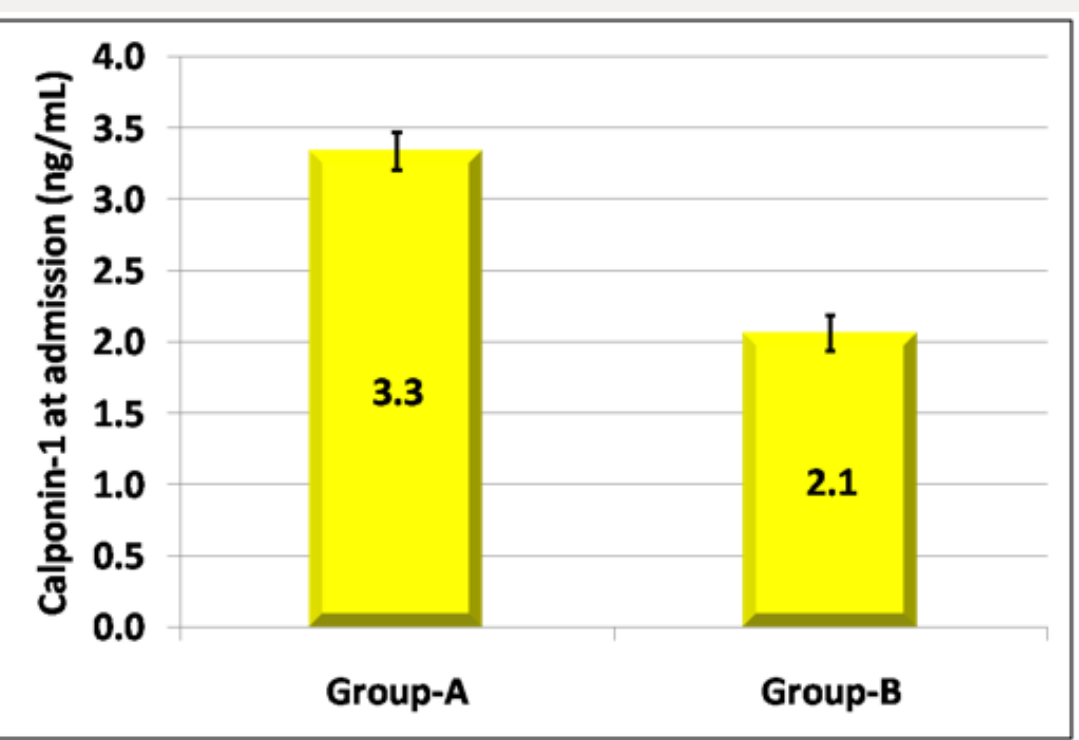

Figure 2: Comparison according to time regarding serum calponin-1 at admissions. 


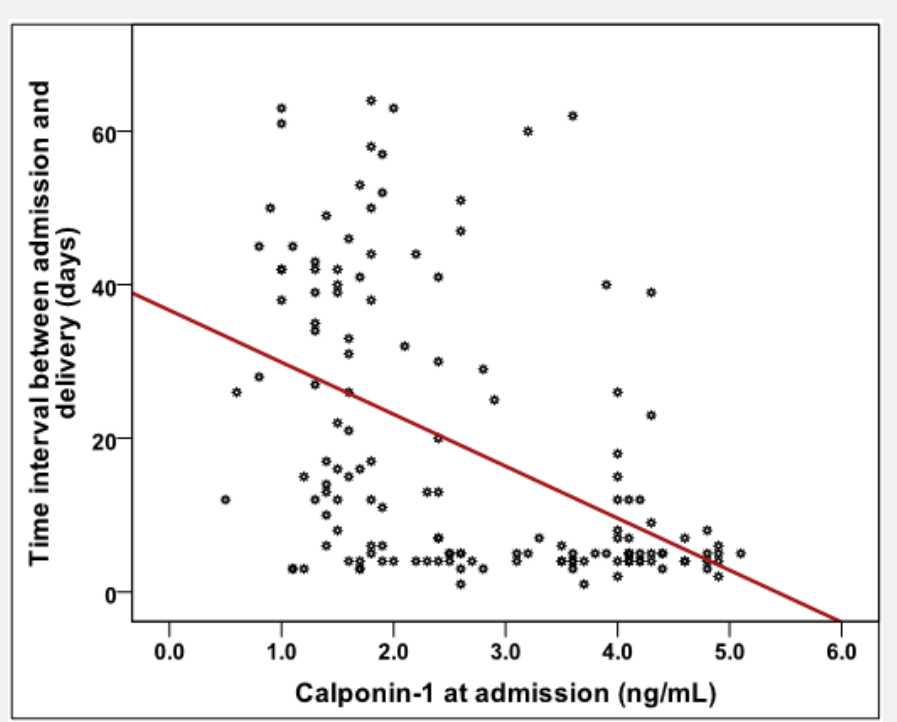

Figure 3: Correlation between time interval between admission and delivery and calponin-1 at admissions.

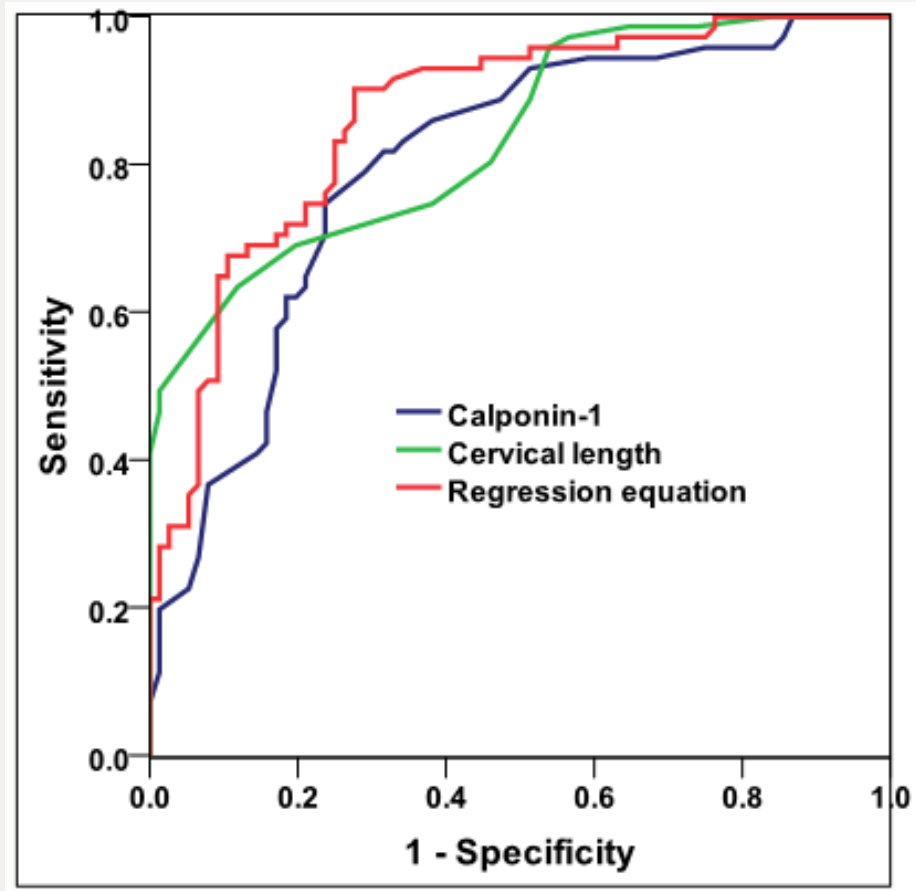

Figure 4: ROC curve for cervical length and calponin-1 at admissions to predict delivery within 1 week from admission.

\section{Statistical Analysis}

The collected data were coded, tabulated, and statistically analyzed using IBM SPSS statistics (Statistical Package for Social Sciences) software version 18.0, IBM Corp., Chicago, USA, 2009. Descriptive statistics were done for quantitative data as minimum\& maximum of the range as well as mean \pm SD (standard deviation) for quantitative data, median, while it was done for qualitative data as number and percentage.

Inferential analyses were done for quantitative variables using independent t-test in cases of two independent groups. In qualitative data, inferential analyses for independent variables were done using Chi square test. While correlations were done using Pearson correlation. Logistic regression model was used to find out independent factors affecting delivery interval. ROC curve was used to evaluate the performance of different tests. The level of significance was taken at $\mathrm{P}$ value $<0.050$ is significant, otherwise is non-significant.

Diagnostic characteristics were calculated as follows:

I. Sensitivity $=($ True positive test $/$ Total positive golden $) \mathrm{x}$ 100

II. Specificity $=($ True negative test $/$ Total negative golden $) \mathrm{x}$ 100 
III. Predictive positive value $=$ (True positive test $/$ Total positive test) $\times 100$

IV. Predictive negative value $=($ True negative test $/$ Total negative test) $\mathrm{x} 100$

V. LR+ = (sensitivity/ 1-specificity $)$

VI. LR- = (1- sensitivity / specificity $)$

VII. Diagnostic accuracy $=$

([True positive test + True negative test] / Total cases) x 100

Youden's index = sensitivity + specificity -1

\section{Discussion}

The current research study displayed that cases that delivered within 1 week from admission had statistically significantly higher CRP, calponin-1, cesarean section and NICU admission as well as statistically significantly lower cervical length at admission, birth weight and APGAR1\& 5. Statistical correlations between Time interval between admission and delivery and cervical length\& calponin- 1 at admissions were significantly positive $(0.387$, $\mathrm{p}<0.001)$ \& significantly negative $(-0.470, \mathrm{p}<0.001)$ respectively while it was between cervical length and calponin-1 at admissions significantly negative $(-0.413, \mathrm{p}<0.001)$. Cervical length and calponin-1 at admissions had moderate Diagnostic performance in predicting delivery within 1 week from admission that not markedly increased if having any of them or by combination in a regression equation. In prior research studies, the chief test for predicting preterm labor was cervical length dimension by applying usage of transvaginal sonography and existence of fetal fibronectin in the cervicovaginal fluid that were considered to be valuable and reliable Clinical markers. various other possible biological markers such as salivary estriol, prolactin in vaginal secretions and interleukin- 6 in fetal amniotic fluid. The investigated biological markers , none of them displayed or revealed clinically hopeful high statistical sensitivity, positive predictive value, negative predictive value. Additionally, they have low diagnostic precision for preterm labor predictability (30-50\%) [7-9] Consequently, various research studies have been performed to display a novel and consistent biological marker which has high diagnostic precision with great value in clinical decision making [10,5,4].

Calponin 1 protein influences contractile actin-myosin filaments in addition to the noncontractile actin cytoskeleton in smooth muscle cells. Prior research studies have revealed that cGMP-dependent protein kinase was statistically significantly reduced in the uterine muscle fibers during pregnancy; and calponin protein was shown to be reallocated to an easily obtainable sub cellular pool. The prior research study findings imply that cGMP-dependent protein kinase does not regulate the quiescence during gestation. Reallocation of thin filament-linked proteins involving calponin 1, on the other hand, could influence smooth uterine muscle contractility or the cytoskeletal cellular structure of myocytes to maintain gestation despite great distention that occurs all intrauterine gestation $[11,12]$. On the other hand, the above -mentioned research study assessed the levels of calponin in uterine tissues; that is not applicable in our research cohort in the current study. Therefore, our research, implied that the calponin 1 escape into the maternal circulation during the lack of normal physiological reallocation in preterm delivery which can be assayed to get valuable data. Finally there are various limitations in the current research study performed .First of all the small sample size used for the study, that could reflect insufficiency in obtained data and results .Future research should consider racial and ethnic differences between patients and should consider variables that could have influence on preterm labor occurrence such as urinary tract infections and medical disorders causing fetal macrosomia such as DM .Future research efforts should consider various biological markers in comparison to reach to the most efficient clinical protocol for predictability of preterm labor that is considered a major clinical issue in obstetrics.

\section{Conclusion}

Measuring and evaluating calponin 1 level in maternal serum could be a useful biological marker in short-range time period for predictability of preterm delivery within patients affected by threatened preterm delivery clinical presentation, besides cervical length dimension measuring.

\section{References}

1. Blencowe H, Cousens S, Oestergaard MZ, Chou D, Moller AB, et al. (2012) National, regional, and worldwide estimates of preterm birth rates in the year 2010 with time trends since 1990 for selected countries: a systematic analysis and implications. Lancet 379: 2162-2172.

2. Sanchez-Ramos L, Delke I, Zamora J, Kaunitz AM (2009) Fetal fibronectin as a short-term predictor of preterm birth in symptomatic patients: a meta-analysis. Obstet Gynecol 114: 631-640.

3. Schaaf JM, Mol BW, Abu-Hanna A, Ravelli AC (2011) Trends in preterm birth: singleton and multiple pregnancies in the Netherlands, 20002007. BJOG 118(10): 1196-1204.

4. Sotiriadis A, Papatheodorou S, Kavvadias A, Makrydimas G (2010) Transvaginal cervical length measurement for prediction of preterm birth in women with threatened preterm labor: a meta-analysis. Ultrasound Obstet Gynecol 35(1): 54-64.

5. Goldenberg RL, Culhane JF, Iams JD, Romero R (2008) Epidemiology and causes of preterm birth. Lancet 371: 75-84.

6. Liu R, Jin JP (2016) Calponin isoforms CNN1, CNN2 and CNN3: Regulators for actin cytoskeleton functions in smooth muscle and non-muscle cells. Gene 585: 143-53.

7. Suzuki T, Distance A, Zizza A, Trimarchi S, Villani M (2008) Preliminary experience with the smooth muscle troponin-like protein, calponin, as a novel biomarker for diagnosing acute aortic dissection. Eur Heart J 29(11): 1439-45.

8. Foster C, Shennan AH (2014) Fetal fibronectin as a biomarker of preterm labor: a review of the literature and advances in its clinical use. Biomark Med 8(4): 471-84.

9. (2012) Practice bulletin no. 130: prediction and prevention of preterm birth. Committee on Practice Bulletins-Obstetrics, The American College of Obstetricians and Gynecologists. Obstet Gynecol 120(4): 964-973. 
10. Dykes AC, Wright GL (2007) Down-regulation of calponin destabilizes actin cytoskeletal structure in A7r5 cells. Can J Physiol Pharmacol 85(2) 225-232.

11. Perlitz Y, Ben-Ami M, Peleg A, Izhaki I, Ben-Shlomo I (2015) Calponin levels in term laboring women. J Matern Fetal Neonatal Med 28(10): $1158-1160$.
12. Huang QQ, Hossain MM, Sun W, Xing L, Pope RM, et al. (2016) Deletion of calponin 2 in macrophages attenuates the severity of inflammatory arthritis in mice. Am J Physiol Cell Physiol 311(4): 673-685.

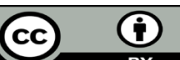

This work is licensed under Creative

Commons Attribution 4.0 License

To Submit Your Article Click Here:

Submit Article

DOI: 10.32474/IGWHC.2018.02.000141

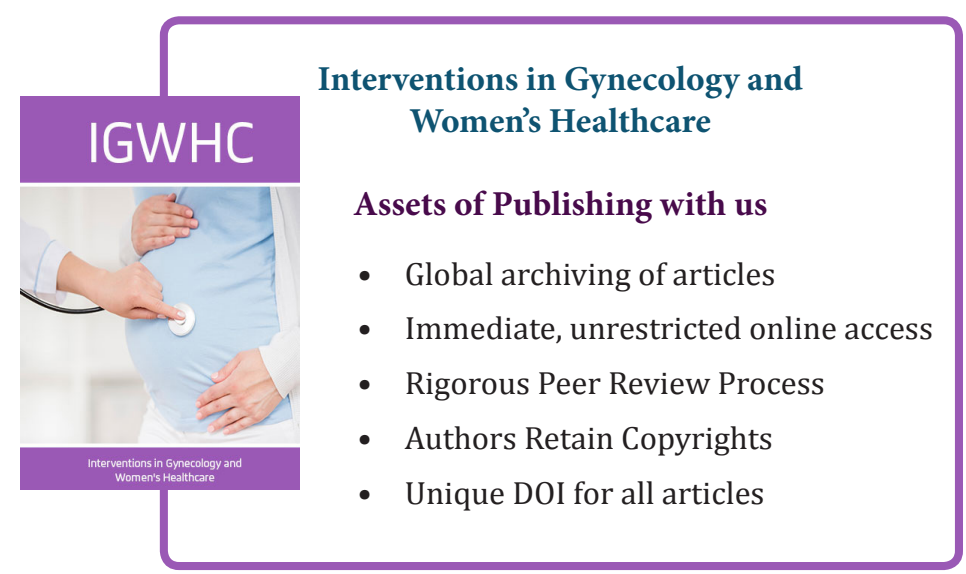

